\title{
Generalized Cesàro summability of Fourier series and its applications
}

\author{
OKTAY DUMAN*
}

ABSTRACT. In this paper, by using generalized Cesàro means based on $q$-integers, we study on approximating continuous and periodic functions by their Fourier series. We also discuss its connection with the concept of statistical convergence. At the end of the paper, some applications and graphical illustrations are also provided.

Keywords: Fourier analysis, Cesàro summability, Fejér's kernel, $q$-integers, statistical convergence.

2020 Mathematics Subject Classification: 42A24, 40G15.

Dedicated to Professor Francesco Altomare, on occasion of his 70th birthday, with esteem and friendship.

\section{INTRODUCTION}

The question of whether the Fourier series of a periodic function converges to the given function is researched by a field known as classical harmonic analysis. It is well-known that convergence is not necessarily given in the general case. However, by using some summability methods, such as Cesàro means and Riesz means, the convergence is possible in some sense (see, for instance, [21]). In the present paper, by using generalized Cesàro means based on $q$ integers (see the next section for details), we study on approximating continuous and periodic functions by their Fourier series.

Let $S_{n}(f)$ denote the partial sums of an integrable and $2 \pi$-periodic function $f$, that is

$$
S_{n}(f ; x)=\frac{a_{0}}{2}+\sum_{k=1}^{n}\left(a_{k} \cos k x+b_{k} \sin k x\right),
$$

where

$$
a_{k}:=\frac{1}{\pi} \int_{-\pi}^{\pi} f(t) \cos k t d t, k=0,1, \ldots
$$

and

$$
b_{k}:=\frac{1}{\pi} \int_{-\pi}^{\pi} f(t) \sin k t d t, k=1,2, \ldots
$$

Then, we may write that

$$
S_{n}(f ; x)=\frac{1}{\pi} \int_{-\pi}^{\pi} f(x+t) D_{n}(t) d t
$$

Received: 10.12.2020; Accepted: 01.02.2021; Published Online: 01.03.2021

*Corresponding author: Oktay Duman; oduman@etu.edu.tr; okitayduman@gmail.com

DOI: $10.33205 / \mathrm{cma} .838606$ 
where $D_{n}(t)$ denotes Dirichlet's kernel given by

$$
D_{n}(t)=\frac{\sin ((n+1 / 2) t)}{2 \sin (t / 2)} .
$$

Furthermore, the classical Cesàro means of $S_{n}(f)$ can be written as follows:

$$
\begin{aligned}
\sigma_{n}(f ; x) & =\frac{S_{0}(f ; x)+S_{1}(f ; x)+\ldots+S_{n}(f ; x)}{n+1} \\
& =\frac{1}{\pi} \int_{-\pi}^{\pi} f(x+t)\left(\frac{1}{n+1} \sum_{k=0}^{n} D_{k}(t)\right) d t \\
& =\frac{1}{\pi} \int_{-\pi}^{\pi} f(x+t) K_{n}(t) d t,
\end{aligned}
$$

where $K_{n}(t)$ denotes Fejér's kernel given by

$$
K_{n}(t)=\frac{\sin ^{2}((n+1 / 2) t)}{2(n+1) \sin ^{2}(t / 2)} .
$$

Now, let $C^{2 \pi}$ denote the space of all continuous and $2 \pi$ periodic functions. Then, it is wellknown that, for any $f \in C^{2 \pi}$, the sequence $\left(\sigma_{n}(f)\right)$ is uniformly convergent to $f$, i.e., $\left(S_{n}(f)\right)$ is uniformly Cesàro summable to $f$.

In order to generalize this summability, we will consider the generalized Cesàro means based on $q$-integers introduced in $[1,4]$.

\section{Q-CESÀRO SUMMABILITY OF FOURIER SERIES}

We first recall some concepts and notation from the $q$-calculus (see [11] for details). For a given $q>0$, the $q$-integer $[n]_{q}$ is given by

$$
[n]_{q}:=1+q+q^{2}+\cdots+q^{n-1} \text { with }[0]_{q}=0 .
$$

Then, for each $n=1,2, \ldots$, we may write that

$$
[n]_{q}=\frac{1-q^{n}}{1-q} \text { for } q \neq 1 .
$$

Now, for a given $q>0$, consider the $q$-Cesàro matrix $C(q)=\left[c_{n k}(q)\right](k, n=0,1,2, \ldots)$ defined by (see $[1,4])$

$$
c_{n k}(q)=\left\{\begin{array}{cc}
\frac{q^{k}}{[n+1]_{q}}, & k=0,1, \ldots, n \\
0, & \text { otherwise. }
\end{array}\right.
$$

Then, we can write the matrix $C(q)$ as follows:

$$
C(q)=\left[\begin{array}{ccccccc}
1 & 0 & 0 & \ldots & 0 & 0 & \ldots \\
\frac{1}{[2]_{q}} & \frac{q}{[2]_{q}} & 0 & \cdots & 0 & 0 & \ldots \\
\frac{1}{[3]_{q}} & \frac{q}{[3]_{q}} & \frac{q^{2}}{[3]_{q}} & \cdots & 0 & 0 & \ldots \\
\vdots & \vdots & \vdots & \vdots & \vdots & \vdots & \ddots \\
\frac{1}{[n+1]_{q}} & \frac{q}{[n+1]_{q}} & \frac{q^{2}}{[n+1]_{q}} & \cdots & \frac{q^{n}}{[n+1]_{q}} & 0 & \ldots \\
\vdots & \vdots & \vdots & \vdots & \vdots & \vdots & \ddots
\end{array}\right] .
$$

Observe that the case of $q=1$ reduces to the classical Cesàro matrix. About regularity of $q$-Cesàro matrix, we can say the following: 
- For any fixed $q \geq 1$, the matrix $C(q)$ is regular (see [1]).

- For a given $0<q<1$, the corresponding matrix $C(q)$ cannot be regular due to the fact that $[n+1]_{q} \rightarrow \frac{1}{1-q}$ as $n \rightarrow \infty$.

- Instead of a fixed $q$, take a sequence $q=\left(q_{n}\right)$ such that the following conditions hold:

$$
0<q_{n}<1 \text { for all } n \in \mathbb{N}_{0}=\{0,1, \ldots\}
$$

and

$$
\lim _{n \rightarrow \infty} q_{n}=1 .
$$

Then, $C(q)$ is still regular. Indeed, from (2.3) and (2.4), we may write that $[n+1]_{q_{n}} \rightarrow \infty$ as $n \rightarrow \infty$ (see, for instance, [17, 18, 19]). Hence, using the well-known SilvermanToeplitz conditions, we immediately get the regularity of $C(q)$ for $q=\left(q_{n}\right)$ (see Example 2.1 for such a sequence).

Because the sequence of partial sums $\left(S_{n}(f)\right)$ need not converge to $f$, we may try looking at their q-Cesàro means as follows:

$$
\begin{aligned}
\sigma_{n}(f ; q ; x) & =\frac{1}{[n+1]_{q}} \sum_{k=0}^{n} q^{k} S_{k}(f ; x) \\
& =\frac{S_{0}(f ; x)+q S_{1}(f ; x)+\ldots+q^{n} S_{n}(f ; x)}{[n+1]_{q}}
\end{aligned}
$$

which implies

$$
\sigma_{n}(f ; q ; x)=\frac{1}{\pi} \int_{-\pi}^{\pi} f(x+t)\left(\frac{1}{[n+1]_{q}} \sum_{k=0}^{n} q^{k} D_{k}(t)\right) d t .
$$

Hence, we may write that

$$
\sigma_{n}(f ; q ; x)=\frac{1}{\pi} \int_{-\pi}^{\pi} f(x+t) K_{n}(q ; t) d t
$$

where

$$
K_{n}(q ; t):=\frac{1}{2[n+1]_{q}} \sum_{k=0}^{n} q^{k} \frac{\sin ((k+1 / 2) t)}{\sin (t / 2)},
$$

say $q$-Fejér's kernel.

We should note that the $q$-Cesàro means in (2.5) may be regarded as a special case of Nörlund or Riesz means of the partial sums of Fourier series. However, it is more convenient to examine the behavior of the corresponding $q$-Fejér's kernel in (2.6) by taking into account the known properties of the $q$-integers. Another important reason for using $q$-integers in this process is that it is possible to weaken the classical limit condition needed in the approximation (see Section 3 for details).

We now start with the fundamental properties of $q$-Fejér's kernel.

Lemma 2.1. Let $q>0$ and $n \in \mathbb{N}_{0}$. Then, we get the followings:

(a) $K_{n}(q ; t)=\frac{(1+q) \sin (t / 2)+q^{n+2} \sin ((n+1 / 2) t)-q^{n+1} \sin ((n+3 / 2) t)}{2[n+1]_{q} \sin (t / 2)\left\{(1-q)^{2} \cos ^{2}(t / 2)+(1+q)^{2} \sin ^{2}(t / 2)\right\}}$.

(b) $K_{n}(q ; t)=\frac{(n+1)}{[n+1]_{q}}\left\{q^{n} K_{n}(t)+\sum_{k=0}^{n-1}\left(q^{k}-q^{k+1}\right) K_{k}(t)\right\}$, where $K_{n}(t)$ is the classical Fejér's kernel given by (1.1). 
(c) $\frac{1}{\pi} \int_{-\pi}^{\pi} K_{n}(q ; t) d t=\frac{n+1}{[n+1]_{q}}$.

(d) If $0<q \leq 1$, then $K_{n}(q ; \cdot) \geq 0$.

Proof. (a) From the definition of $K_{n}(q ; t)$ in $(2.6)$, we may write that

$$
\begin{aligned}
K_{n}(q ; t) & =\frac{1}{2[n+1]_{q} \sin (t / 2)} \operatorname{Im}\left\{\sum_{k=0}^{n} q^{k} e^{i(k+1 / 2) t}\right\} \\
& =\frac{1}{2[n+1]_{q} \sin (t / 2)} \operatorname{Im}\left\{e^{i t / 2} \sum_{k=0}^{n}\left(q e^{i t}\right)^{k}\right\} \\
& =\frac{1}{2[n+1]_{q} \sin (t / 2)} \operatorname{Im}\left\{e^{i t / 2} \frac{1-q^{n+1} e^{i(n+1) t}}{1-q e^{i t}}\right\} \\
& =\frac{1}{2[n+1]_{q} \sin (t / 2)} \operatorname{Im}\left\{\frac{1-q^{n+1} e^{i(n+1) t}}{e^{-i t / 2}-q e^{i t / 2}}\right\}
\end{aligned}
$$

Observe that

$$
\operatorname{Im}\left\{\frac{1-q^{n+1} e^{i(n+1) t}}{e^{-i t / 2}-q e^{i t / 2}}\right\}=\frac{A_{n}(q, t)-B_{n}(q, t)}{(1-q)^{2} \cos ^{2}(t / 2)+(1+q)^{2} \sin ^{2}(t / 2)},
$$

where

$$
\begin{aligned}
& A_{n}(q, t)=(1+q)\left(1-q^{n+1} \cos ((n+1) t)\right) \sin (t / 2), \\
& B_{n}(q, t)=(1-q) q^{n+1} \sin ((n+1) t) \cos (t / 2) .
\end{aligned}
$$

Hence, using some appropriate trigonometric identities, we obtain that

$$
\begin{aligned}
A_{n}(q, t)-B_{n}(q, t)= & (1+q) \sin (t / 2)+q^{n+2} \sin ((n+1 / 2) t) \\
& -q^{n+1} \sin ((n+3 / 2) t),
\end{aligned}
$$

which immediately gives the equality in $(a)$.

(b) If we use Abel's partial sums identity in (2.6), then we observe from (1.1) that

$$
\begin{aligned}
K_{n}(q ; t) & =\frac{(n+1)}{[n+1]_{q}} \sum_{k=0}^{n} q^{k} \frac{\sin ((k+1 / 2) t)}{2(n+1) \sin (t / 2)} \\
& =\frac{(n+1)}{[n+1]_{q}}\left\{q^{n} K_{n}(t)+\sum_{k=0}^{n-1}\left(q^{k}-q^{k+1}\right) K_{k}(t)\right\},
\end{aligned}
$$

which completes the proof of $(b)$.

(c) We may write from $(b)$ that

$$
\begin{aligned}
& \frac{1}{\pi} \int_{-\pi}^{\pi} K_{n}(q ; t) d t \\
= & \frac{n+1}{[n+1]_{q}}\left\{q^{n}\left(\frac{1}{\pi} \int_{-\pi}^{\pi} K_{n}(t) d t\right)+(1-q) \sum_{k=0}^{n-1} q^{k}\left(\frac{1}{\pi} \int_{-\pi}^{\pi} K_{k}(t) d t\right)\right\} .
\end{aligned}
$$


Since $\frac{1}{\pi} \int_{-\pi}^{\pi} K_{n}(t) d t=1$, we see that

$$
\begin{aligned}
\frac{1}{\pi} \int_{-\pi}^{\pi} K_{n}(q ; t) d t & =\frac{n+1}{[n+1]_{q}}\left\{q^{n}+(1-q) \sum_{k=0}^{n-1} q^{k}\right\} \\
& =\frac{n+1}{[n+1]_{q}}\left\{q^{n}+(1-q)[n]_{q}\right\} \\
& =\frac{n+1}{[n+1]_{q}}
\end{aligned}
$$

which gives $(c)$.

$(d)$ It is clear from $(b)$, since $0<q \leq 1$.

Remark 2.1. If one takes $q=1$ in Lemma 2.1 then (a) implies

$$
\begin{aligned}
K_{n}(1 ; t) & =\frac{2 \sin (t / 2)+\sin ((n+1 / 2) t)-\sin ((n+3 / 2) t)}{8(n+1) \sin ^{3}(t / 2)} \\
& =\frac{1-\cos ((n+1) t)}{4(n+1) \sin ^{2}(t / 2)} \\
& =\frac{\sin ^{2}((n+1) t / 2)}{2(n+1) \sin ^{2}(t / 2)} \\
& =K_{n}(t),
\end{aligned}
$$

and $(b)$ implies the same equality $K_{n}(1 ; t)=K_{n}(t)$, and also $(c)$ and $(d)$ implies the classical results $\frac{1}{\pi} \int_{-\pi}^{\pi} K_{n}(t) d t=1$ and $K_{n}(\cdot) \geq 0$, respectively.

Theorem 2.1. Assume that the sequence $q=\left(q_{n}\right)$ satisfies the conditions (2.3) and (2.4). Then, for the operators in (2.5), we get

$$
\lim _{n \rightarrow \infty} \sigma_{n}\left(f ; q_{n} ; x\right)=f(x) \text { uniformly with respect to } x
$$

for every $f \in C^{2 \pi}$.

Proof. Since the operators in (2.5) are positive and linear, from the well-known Korovkin theorem for $2 \pi$-periodic continuous functions (see $[2,12])$, it is enough to show that

$$
\sigma_{n}\left(f_{i} ; q_{n} ; x\right) \rightrightarrows f_{i}(x) \text { for } i=0,1,2,
$$

where $f_{0}(x)=1, f_{1}(x)=\sin x$ and $f_{2}(x)=\cos x$. As usual, the symbol $\rightrightarrows$ denotes the uniform convergence. Now, it is easy to check that

$$
\sigma_{n}\left(f_{0} ; q_{n} ; x\right)=f_{0}(x)=1 \text {. }
$$

From the definition of the operators, we observe that

$$
\begin{aligned}
\sigma_{n}\left(f_{1} ; q_{n} ; x\right) & =\frac{S_{0}\left(f_{1} ; x\right)+q_{n} S_{1}\left(f_{1} ; x\right)+q_{n}^{2} S_{2}\left(f_{1} ; x\right)+\cdots+q_{n}^{n} S_{n}\left(f_{1} ; x\right)}{[n+1]_{q_{n}}} \\
& =\frac{0+q_{n} \sin x+q_{n}^{2} \sin x+\cdots+q_{n}^{n} \sin x}{[n+1]_{q_{n}}} \\
& =\frac{q_{n}+q_{n}^{2}+\cdots+q_{n}^{n}}{[n+1]_{q_{n}}} \sin x,
\end{aligned}
$$

which implies

$$
\sigma_{n}\left(f_{1} ; q_{n} ; x\right)=\left(1-\frac{1}{[n+1]_{q_{n}}}\right) \sin x .
$$




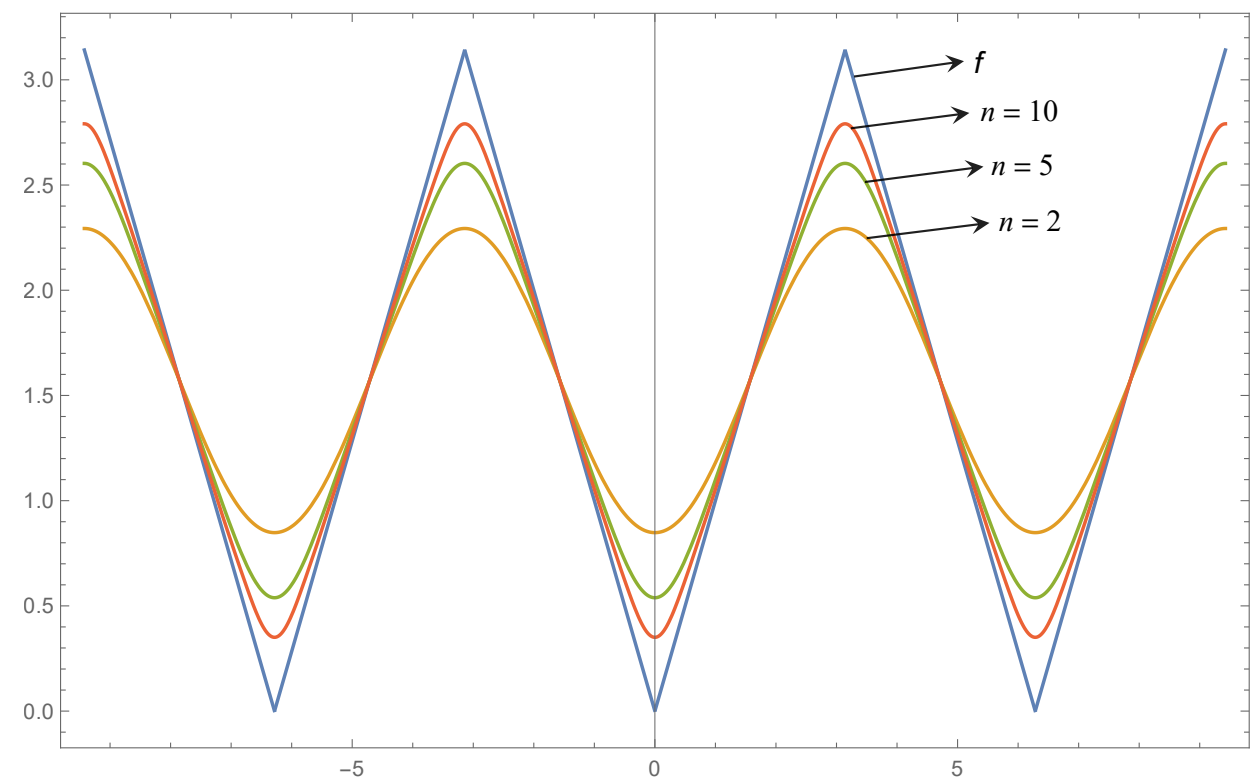

FIGURE 1. $q$-Cesàro approximation to the function $f(x)=|x|$ by the operators $\sigma_{n}\left(f ; q_{n} ; x\right)$ associated with the sequence $q=\left(q_{n}\right)$ given by (2.11)

Similarly, we also get

$$
\sigma_{n}\left(f_{2} ; q_{n} ; x\right)=\left(1-\frac{1}{[n+1]_{q_{n}}}\right) \cos x .
$$

Taking limit as $n \rightarrow \infty$ in (2.8), (2.9) and (2.10) and also considering the assumptions (2.3) and (2.4), we obtain (2.7), which completes the proof.

Example 2.1. Define the function $f$, for $x \in[-\pi, \pi]$, by $f(x)=|x|$ and extend its domain periodically to the whole real line which coincides on $[-\pi, \pi]$. Consider the sequence $q=\left(q_{n}\right)$ given by

$$
q_{n}=1-\frac{1}{n+2} .
$$

Then, all conditions of Theorem 2.1 holds, which implies

$$
\lim _{n \rightarrow \infty} \sigma_{n}\left(f ; q_{n} ; x\right)=f(x)
$$

uniformly with respect to $x$. This (uniform) q-Cesàro summability is indicated in Figure 1 with the parameter values $n=2,5,10$.

Example 2.2. Consider the $2 \pi$-periodic and even function $f$ defined on $[0, \pi]$ by

$$
f(x)=\sum_{k=1}^{\infty} \frac{1}{k^{2}} \sin \left(\left(2^{k^{3}}+1\right) \frac{x}{2}\right) .
$$

Then, according to Weierstrass M-test, we get the continuity of $f$ on $\mathbb{R}$. Hence, Theorem 2.1 implies that, for any sequence $q=\left(q_{n}\right)$ satisfying $(2.3)$ and $(2.4), \lim _{n \rightarrow \infty} \sigma_{n}\left(f ; q_{n} ; x\right)=f(x)$ uniformly with respect to $x$. However, one can observe that the classical partial sums of the function $f$ in (2.12) cannot converge to $f$ at the origin. More precisely, the sequence $\left(S_{n}(f ; 0)\right)$ diverges to the infinity as $n \rightarrow \infty$ (see, for instance, [20]). 


\section{EXTENSION TO THE STATISTICAL CONVERGENCE AND CONCLUDING REMARKS}

In this section, we will work on the situation where the limit condition in (2.4) is weakened. For example, we can consider the concept of statistical convergence (see [8] by Fast). We note that this type of convergence has been introduced a few years earlier by Zygmund with the name "almost convergence" (see [21, Vol. II, Chap. XIII]). Later on, the statistical convergence has been frequently used not only in the summability theory, but also in the approximation theory (see $[3,6,7,10,13,14,15,16])$. We recall that the (asymptotic) density, $\delta(K)$, of a set $K \subset \mathbb{N}$ is defined by

$$
\delta(K):=\lim _{n \rightarrow \infty} \frac{1}{n+1} \#\{0 \leq k \leq n: k \in K\}
$$

provided that the limit exists, where the symbol \# denotes the cardinal number of a set. Using this density, a sequence $\left(x_{n}\right)$ is said to be statistically convergent to a number $L$, denoted by $s t-\lim _{n \rightarrow \infty} x_{n}=L$, if for every $\varepsilon>0$,

$$
\delta\left(\left\{0 \leq k \leq n:\left|x_{k}-L\right| \geq \varepsilon\right\}\right)=0,
$$

that is

$$
\lim _{n \rightarrow \infty} \frac{1}{n+1} \#\left\{0 \leq k \leq n:\left|x_{k}-L\right| \geq \varepsilon\right\}=0 .
$$

It is well-known that every convergent sequence is statistically convergent to the same value, but the converse is not always true. Furthermore, for a given sequence $\left(x_{n}\right)$, st $-\lim _{n \rightarrow \infty} x_{n}=L$ if and only if there exists an index set $K=\left\{k_{n}: n \in \mathbb{N}_{0}\right\}$ of density 1 such that the subsequence $\left(x_{k_{n}}\right)$ converges to $L$ (in the usual sense) as $n \rightarrow \infty$ (see [5,9] for further properties of statistical convergence).

For a given sequence $q=\left(q_{n}\right)$ with $0<q_{n}<1$, we replace the limit condition (2.4) with the following weaker condition:

$$
s t-\lim _{n \rightarrow \infty} q_{n}=1 .
$$

In this case, the corresponding $q$-Cesàro matrix in (2.2) does not need to be regular. For example, consider the sequence $q=\left(q_{n}\right)$ defined by

$$
q_{n}= \begin{cases}\frac{1}{2}\left(1-\frac{1}{n+2}\right), & \text { if } n=m^{2}(m=0,1, \ldots) \\ 1-\frac{1}{n+1}, & \text { otherwise. }\end{cases}
$$

Observe that, in this case, $\left[m^{2}+1\right]_{q_{m^{2}}} \rightarrow 2$ as $m \rightarrow \infty$. Despite this negative situation, we obtain the following statistical approximation theorem.

Theorem 3.2. Assume that the sequence $q=\left(q_{n}\right)$ satisfy the conditions (2.3) and (3.13). Then, for the operators in (2.5), we get

$$
s t-\lim _{n \rightarrow \infty} \sigma_{n}\left(f ; q_{n} ; x\right)=f(x) \text { uniformly with respect to } x
$$

for every $f \in C^{2 \pi}$.

Proof. This immediately follows from the statistical Korovkin theorem for periodic functions (see [6]) since, for each $i=0,1,2$,

$$
s t-\lim _{n \rightarrow \infty} \sigma_{n}\left(f_{i} ; q_{n} ; x\right)=f_{i}(x) \text { uniformly with respect to } x,
$$

where $f_{i}$ are the test functions stated before. 


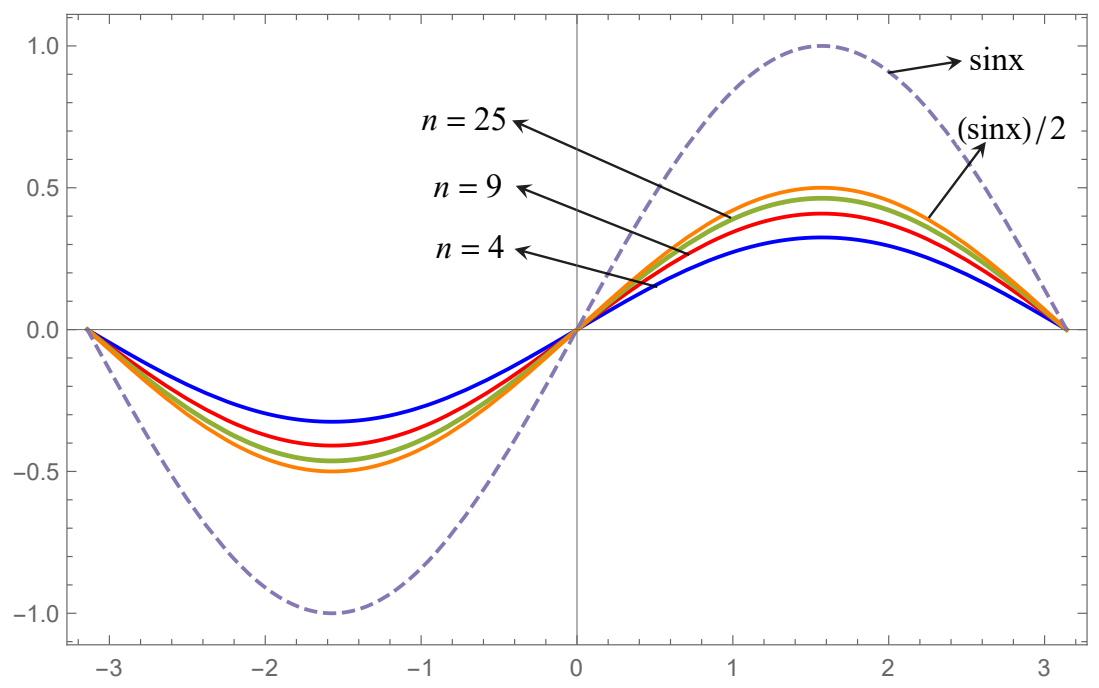

FIGURE 2. $q$-Cesàro approximation to the function $f_{1}(x)=\sin x$ by $\left(\sigma_{n}\left(f_{1} ; q_{n} ; x\right)\right)$ associated with the sequence $q=\left(q_{n}\right)$ given by (3.14) fails for the values $n=m^{2}$

Remark 3.2. We know from Example 2.2 that there exists a function $f$ in $C^{2 \pi}$ such that approximation to $f$ by the partial sums $\left(S_{n}(f)\right)$ fails. Now consider the sequence $q=\left(q_{n}\right)$ given by $(3.14)$. Then, using the test function $f_{1}(x)=\sin x$, we get

$$
\sigma_{m^{2}}\left(f_{1} ; q_{m^{2}} ; x\right)=\left(1-\frac{1}{\left[m^{2}+1\right]_{q_{m^{2}}}}\right) \sin x \rightarrow \frac{\sin x}{2}(\text { as } m \rightarrow \infty),
$$

which is indicated in Figure 2 for some values $n=m^{2}$. Hence, for the sequence $q=\left(q_{n}\right)$ in $(3.14)$, $q$-Cesàro approximation in Theorem 2.1 fails either. However, one can obtain from Theorem 3.2 that (3.15) holds for any function in $C^{2 \pi}$. This situation is indicated in Figure 3 for some values $n \neq m^{2}$, where the set of all nonnegative integers $n$ satisfying $n \neq m^{2}$ has density one. 


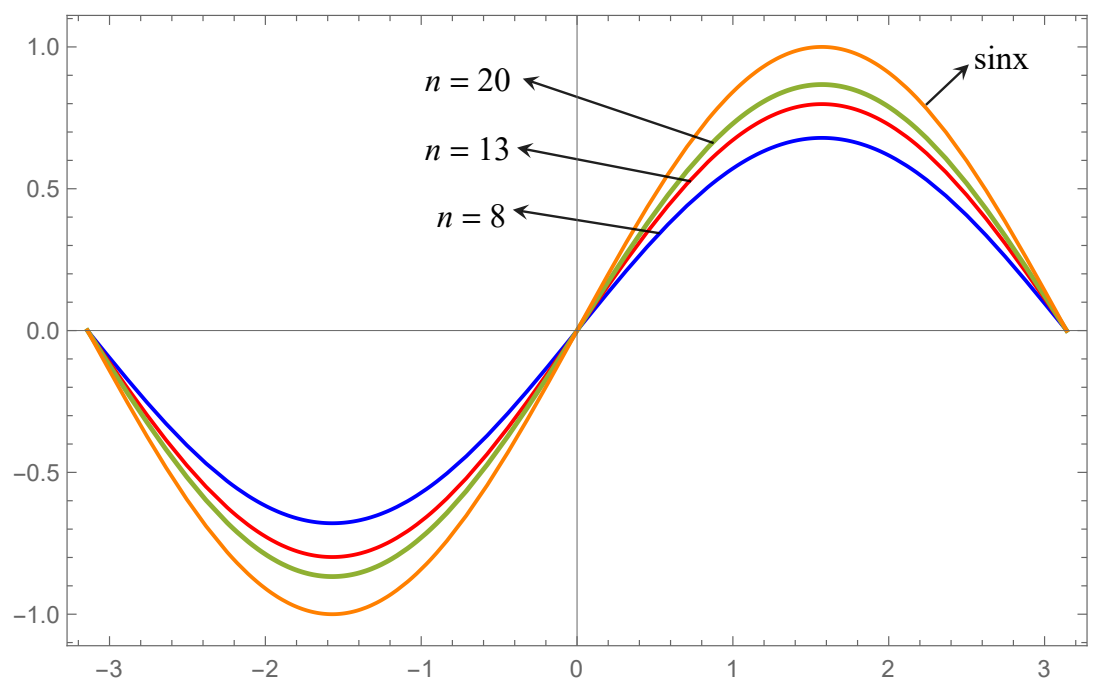

FIGURE 3. Statistical $q$-Cesàro approximation to the function $f_{1}(x)=\sin x$ by $\left(\sigma_{n}\left(f_{1} ; q_{n} ; x\right)\right)$ associated with the sequence $q=\left(q_{n}\right)$ given by (3.14) for the values $n \neq m^{2}$

\section{REFERENCES}

[1] H. Aktuglu, S. Bekar: q-Cesàro matrix and q-statistical convergence, J. Comput. Appl. Math., 235 (16) (2011), 47174723.

[2] F. Altomare, M. Campiti: Korovkin-type approximation theory and its applications, De Gruyter Studies in Mathematics, 17. Walter de Gruyter \& Co., Berlin, (1994).

[3] G. A. Anastassiou, O. Duman: Towards intelligent modeling: statistical approximation theory, Intelligent Systems Reference Library, 14. Springer-Verlag, Berlin, (2011).

[4] J. Bustoz, L. F. Gordillo: q-Hausdorff summability, J. Comput. Anal. Appl., 7 (1) (2005), 35-48.

[5] J. S. Connor: The statistical and strong p-Cesàro convergence of sequences, Analysis, 8 (1-2) (1988), 47-63.

[6] O. Duman: Statistical approximation for periodic functions, Demonstratio Math., 36 (4) (2003), 873-878.

[7] O. Duman, M. K. Khan and C. Orhan: A-statistical convergence of approximating operators, Math. Inequal. Appl., 6 (4) (2003), 689-699.

[8] H. Fast: Sur la convergence statistique, Colloq. Math., 2 (1951), 241-244.

[9] J. A. Frid: On statistical convergence Analysis, 5 (4) (1985), 301-313.

[10] A. D. Gadjiev, C. Orhan: Some approximation theorems via statistical convergence, Rocky Mountain J. Math., 32 (1) (2002), 129-138.

[11] V. Kac, P. Cheung: Quantum calculus, Universitext. Springer-Verlag, New York, (2002).

[12] P. P. Korovkin: Linear operators and approximation theory, Translated from the Russian ed. (1959). Russian Monographs and Texts on Advanced Mathematics and Physics, Vol. III. Gordon and Breach Publishers, Inc., New York; Hindustan Publishing Corp., Delhi, India, (1960).

[13] F. Móricz: Statistical convergence of multiple sequences Arch. Math. (Basel), 81 (1) (2003), 82-89.

[14] F. Móricz: Statistical convergence of Walsh-Fourier series, Acta Math. Acad. Paedagog. Nyházi. (N.S.), 20 (2) (2004), $165-168$.

[15] F. Móricz: Statistical convergence of sequences and series of complex numbers with applications in Fourier analysis and summability, Anal. Math., 39 (4) (2013), 271-285.

[16] F. Móricz: Strong Cesàro $|C, 1,1|$ summability and statistical convergence of double orthogonal series, Anal. Math., 43 (1) (2017), 103-116.

[17] H. Oruc, G. M. Phillips: A generalization of the Bernstein polynomials, Proc. Edinburgh Math. Soc. (2), 42 (2) (1999), 403-413.

[18] G. M. Phillips: A survey of results on the q-Bernstein polynomials, IMA J. Numer. Anal., 30 (1) (2010), 277-288. 
[19] G. M. Phillips: On generalized Bernstein polynomials. Numerical analysis, 263-269, World Sci. Publ., River Edge, NJ, (1996).

[20] Webpage: https://www.mathcounterexamples.net/continuous-function-with-divergent-fourier-series

[21] A. Zygmund: Trigonometric series, Vol. I and II. Third edition. Cambridge Mathematical Library. Cambridge University Press, Cambridge, (2002).

\section{OKTAY DUMAN}

TOBB ECONOMICS AND TECHNOLOGY UNIVERSITY

DEPARTMENT OF MATHEMATICS

SÖĞÜTÖZÜ TR-06530, ANKARA, TURKEY

ORCID: 0000-0001-7779-6877

E-mail address: oduman@etu.edu.tr; okitayduman@gmail.com 